\title{
Automatic Guidance of an Ultrasound Probe by Visual Servoing Based on B-Mode Image Moments
}

\author{
Rafik Mebarki, Alexandre Krupa, and Christophe Collewet \\ IRISA, INRIA Rennes-Bretagne Atlantique, 35042 Rennes, France \\ \{Rafik.Mebarki, Alexandre.Krupa, Christophe.Collewet\}@irisa.fr
}

\begin{abstract}
We propose a new visual servo approach to automatically control in real-time the full motion of a $2 \mathrm{D}$ ultrasound (US) probe held by a medical robot in order to reach a desired image of motionless soft tissue object in B-mode ultrasound imaging. Combinations of image moments of the observed object cross-section are used as feedback information in the visual control scheme. These visual features are extracted in realtime from the US image thanks to a fast image segmentation method. Simulations performed with a static US volume containing an egg-shaped object, and ex-vivo experiments using a robotized US probe that interacts with a motionless rabbit heart immersed in water, show the validity of this new approach and its robustness to different perturbations. This method shows promise for a variety of US-guided medical interventions that require real-time servoing.
\end{abstract}

\section{Introduction}

Ultrasound (US) 2D imaging has great potential in a wide range of robot-assisted interventions due to its non-invasiveness, real-time images streaming, and low cost. However the direct use of the US imaging information in robot control is a big challenge due to the high image noise level and has been hardly considered over the past years. In [1], a robot-assisted system designed for 3D US imaging 22 is controlled by US visual servoing in order to center the section of an artery within the 2D US image. However, only the in-plane motions (3 DOF) of the probe corresponding to the two translations and the rotation in the US image plane are controlled directly from the image, while the other 3 DOF are teleoperated by the user. In [3], a 5 DOF medical robot to perform real-time US-guided needle insertion for percutaneous cholecystostomy is presented. However, only two DOF are controlled to position the needle which is mechanically constrained to lie in the motionless probe observation plane. In these two prior works, visual servoing was only performed for the probe in-plane motions thanks to a simple model of the probe interaction which in this case does not require any $3 \mathrm{D}$ information of the observed object of interest. However, if the visual task consists also in controlling the out-of-plane motions, these previous methods cannot be used and it is necessary to model the complete interaction between the probe 
and the object. In fact, the standard visual servoing methods are devoted only for the case of a camera that provides a projection of the $3 \mathrm{D}$ world to a $2 \mathrm{D}$ image and not for the use of an US probe that provides full information only in its $2 \mathrm{D}$ observation plane.

Some recent research works dealt with out-of-plane motion servoing by modeling the interaction of the US probe with the considered object. In [4, two image points corresponding to the intersections of a laparoscopic instrument forceps with the US plane are used to servo the 4 DOF of the instrument. In [5], an US image-based visual servoing allows to control the motion of an US robotized probe in order to reach a desired image section of an egg-shaped object. In this latter work, the object image edge was modelled by a third order polynomial whose coefficients were selected as feedback features in the control law. However, these coefficients have no physical meaning. Moreover, they are very sensitive to image noise and limit the use of the method to specific object shapes. More recently, in [6] a new visual servoing approach relying on the correlation of ultrasound speckle contained in the B-mode US images has been proposed to stabilize an US image of moving soft tissue without the need of segmentation of structures of interest. Nevertheless this method is entirely different as the one we propose here and cannot be used to reach a desired image from an initial B-scan.

In this work, we present a system to fully automatically and in real time position an US probe held by a medical robot in order to reach a target US crosssection image by means of the image moments. This method can be exploited for many applications. For example, for pathology analysis it would be helpful to automatically and accurately position the US probe in order to obtain a $2 \mathrm{D}$ cross-section image of a tumor having a maximum similarity with a previous one derived from a pre-operative 3D imaging that was performed with the same (US) or other imaging modalities (MRI, CT-SCAN). Also, during a biopsy or a radio frequency ablation procedure, reaching and maintaining automatically a desired cross-section by the US probe would be very helpful for the surgeon. In our approach, the visual features that we propose to use in the control law are combinations of the image moments of the observed object cross-section since they are generic with intuitive and geometric meaning and are robust with respect to measurement perturbations. The paper is organized as follows: Section 2 briefly introduces the visual servo control approach based on US image moments. Section 3 presents the real-time method used to extract the object cross-section contour from the US images. Simulations and experimental results are presented and discussed in Section 4

\section{Visual Servoing}

The robotic task consists in automatically moving an US probe actuated by a medical robot in order to reach a previously learned US B-scan image. In our approach, we propose to use the US image moments as feedback to the control system. This feedback information is represented by the visual features vector $\mathbf{s}$ that we choose as follows: $\mathbf{s}=\left(x_{g}, y_{g}, \alpha, a, l_{1}\right)$ where $\left(x_{g}, y_{g}\right), \alpha, a, l_{1}$ are 
respectively the gravity center, main orientation, area and the main axis length of the object cross-section observed in the US image. They are defined in function of the image moments as follows:

$$
a=m_{00}, x_{g}=m_{10} / a, y_{g}=m_{01} / a, \alpha=\frac{1}{2} \arctan \left(\frac{2 \mu_{11}}{\mu_{20}+\mu_{02}}\right),
$$

$l_{1}=\frac{2}{a}\left(\mu_{02}+\mu_{20}+\sqrt{\left(\mu_{20}-\mu_{02}\right)^{2}+4 \mu_{11}^{2}}\right)$ where $m_{i j}$ denotes the image moments of order $i+j$ defined by:

$$
m_{i j}=\iint_{\mathcal{S}} f(x, y) d x d y
$$

with $f(x, y)=x^{i} y^{j}$ and where $(x, y)$ represent the image coordinates of a point belonging to the image cross-section $\mathcal{S}$ of the object observed in the US image (see Fig. 1(a). Here $\mu_{11}, \mu_{20}$ and $\mu_{02}$ are the centered moments of order 2. Note that (1) requires a segmented image to be computed.

To design the visual control scheme it is required to determine the interaction matrix $\mathbf{L}_{\mathbf{s}}$ relating the time variation $\dot{\mathbf{s}}$ of the visual features $\mathbf{s}$ to the probe velocity $\mathbf{v}=\left(v_{x}, v_{y}, \omega_{z}, v_{z}, \omega_{x}, \omega_{y}\right)$ in the linear form $\dot{\mathbf{s}}=\mathbf{L}_{\mathbf{s}} \mathbf{v}$ (see [7]). Here, $\boldsymbol{v}=\left(v_{x}, v_{y}, v_{z}\right)$ and $\boldsymbol{\omega}=\left(\omega_{x}, \omega_{y}, \omega_{z}\right)$ are respectively the translational and the rotational velocity of the US probe. As $\dot{\mathbf{s}}$ is directly linked to the image moments variation $\dot{m}_{i j}$ we need to relate $\dot{m}_{i j}$ in function of $\mathbf{v}$. By performing time derivative on (11) and then applying the Green's theorem in order to express $\dot{m}_{i j}$ as a contour integral on the image section edge $\mathcal{C}$, we obtain:

$$
\dot{m}_{i j}=-\oint_{\mathcal{C}}[f(x, y) \dot{y}] d x+\oint_{\mathcal{C}}[f(x, y) \dot{x}] d y
$$

where $(\dot{x}, \dot{y})$ are the image coordinates time variation of a given point belonging to the edge $\mathcal{C}$ (see Fig. 1(a). Therefore, $\mathbf{L}_{\mathbf{s}}$ is obtained by relating the image point variation $(\dot{x}, \dot{y})$ to $\mathbf{v}$. This interaction was modelled in our previous work 8] were we used the fact that, locally, each edge point moves on the plane $\pi$ that is tangent to the organ surface at the considered point position as depicted in Fig. 1(a), All calculus done, the interaction matrix is finally given by:

$$
\mathbf{L}_{\mathbf{s}}=\left[\begin{array}{cccccc}
-1 & 0 & y_{g} & x_{g v z} & x_{g \omega x} & x_{g \omega y} \\
0 & -1 & -x_{g} & y_{g v z} & y_{g \omega x} & y_{g \omega y} \\
0 & 0 & -1 & \alpha_{v z} & \alpha_{\omega x} & \alpha_{\omega y} \\
0 & 0 & 0 & a_{v z} & a_{\omega x} & a_{\omega y} \\
0 & 0 & 0 & l_{1 v z} & l_{1 \omega x} & l_{1 \omega y}
\end{array}\right]
$$

We can notice that $a$ and $l_{1}$ are invariants to in-plane motions of the probe as shown by the zero components of the first three columns of $\mathbf{L}_{\mathbf{s}}$ and therefore seems to be good features for out-of-plane motion control whereas $x_{g}, y_{g}$ and $\alpha$ are well adapted for in-plane motion control, with a good decoupling property that can be seen by referring to the triangular part of $\mathbf{L}_{\mathbf{s}}$. The coefficients involved in the last three columns relating the probe out-of plane motion are 


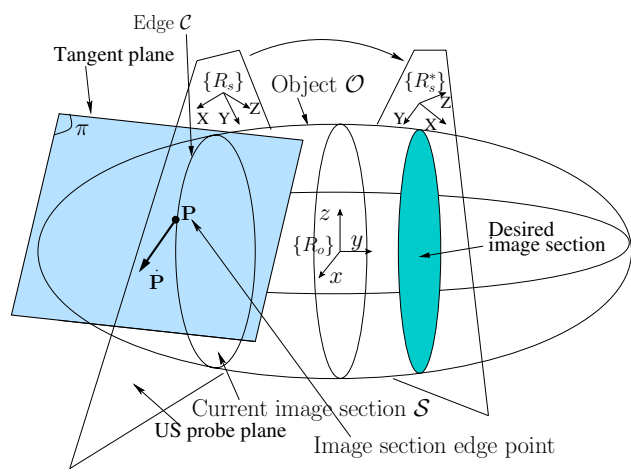

(a)

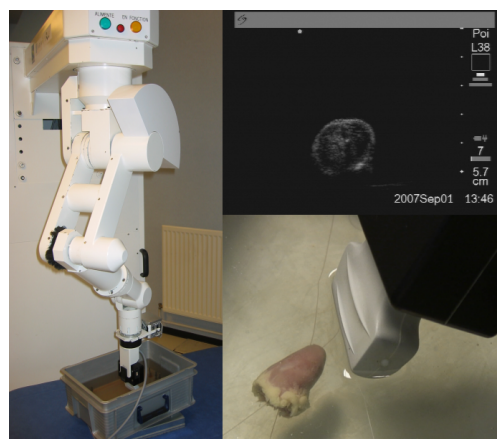

(b)

Fig. 1. (a) Interaction between the ultrasound probe and the object - (b) Experimental setup: (left) ultrasound probe mounted on a 6 DOF medical robot - (right) Observed US image and the rabbit heart suspended in a water-filled box

function of the image moments and some 3D parameters representing the organ geometrical shape. Let us point out that our approach is generic and can be used for complex object shapes whose geometrical parameters are estimated from pre-operative imaging. To automatically move the probe we use the classical control law (see [7]): $\mathbf{v}=-\lambda \hat{\mathbf{L}}_{\mathbf{s}}^{+}\left(\mathbf{s}-\mathbf{s}^{*}\right)$, where $\lambda$ is a positive gain, $\mathbf{s}^{*}$ is the visual features vector of the learned desired US image, and $\hat{\mathbf{L}}_{\mathbf{s}}^{+}$is the pseudo inverse of an estimation of the interaction matrix $\mathbf{L}_{\mathbf{s}}$.

\section{Image Processing}

The visual features defined in the previous Section are based on the computation of the image moments given by (10). However, as already mentioned, this computation requires a segmented image so that $\mathcal{C}$ can be precisely estimated. This can be done by active contours. Indeed, since their first introduction [9], they have proven to be very powerful in various contexts, including segmentation, edge detection and target tracking.

Active contours are deformable curves which are defined in an image domain and move under the influence of internal forces coming from the curve itself and external forces generated from the image (typically gradient information for edge detection). Numerous approaches are reported in the literature. Nevertheless, they can be classified into two main categories: geometric snakes [1011] where the curves is described from a level set representation, or parametric snakes where either the curve is described as a discrete collection of points 912 or described by an appropriate parametric function such as B-splines for example 13 14. However, when considering visual servoing issues, we have to ensure a high acquisition rate (in order to not penalize the dynamics of the robot and the stability of the control scheme). Thus, the image processing duration has to be as near as possible to the video rate (40 ms). Consequently, our approach is 


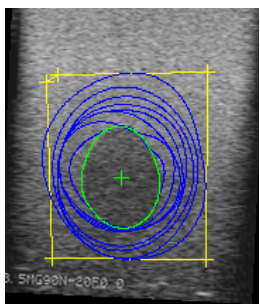

(a)

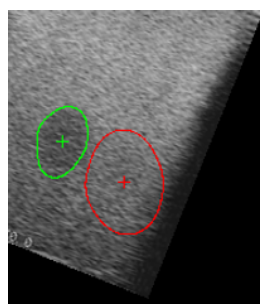

(b)

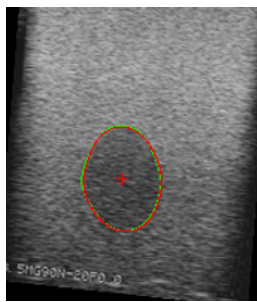

(c)

Visual features errors

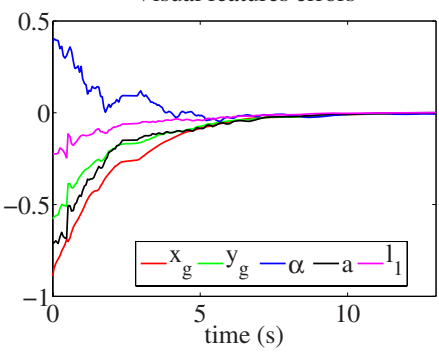

(d)

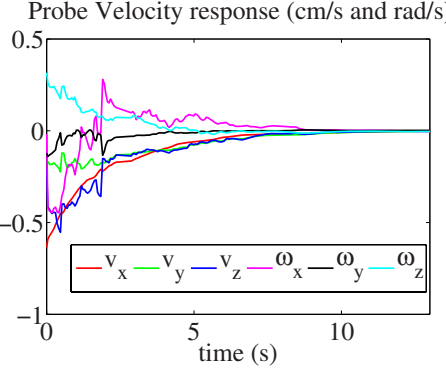

(e)

Fig. 2. (a) Convergence of the active contour on the desired image (yellow: initial curve, blue: motion of the curves to reach the desired contour, green: convergence of the curve) - Results from the US simulator: (b) initial (green) and desired (red) image sections edges before applying visual servoing, (c) current (green) and desired (red) image sections edges after applying visual servoing, (d) visual error response $(\mathrm{cm}, \mathrm{cm}$, $\mathrm{rad}, \mathrm{cm}^{2}$ and $\left.\mathrm{cm}\right),(\mathrm{e})$ velocity applied to the probe

based on parametric snakes since it is well known that geometric approaches are time consuming [15. Thus we present here a fast parametric approach.

It is based on a polar description of the contour of a curve $\mathcal{C}_{\mathbf{q}}$ parameterized by a vector q:

$$
\mathcal{C}_{\mathbf{q}}(u): \mathbf{x}(u)=\mathbf{x}_{g}+\rho_{\mathbf{q}}(u)\left(\begin{array}{c}
\cos u \\
\sin u
\end{array}\right)
$$

with $u \in[0,2 \pi]$. Besides, we perform a Fourier expansion of the radius $\rho_{\mathbf{q}}(u)$ :

$$
\rho_{\mathbf{q}}(u)=q_{0}+\sum_{k=1}^{k=h} q_{k} \cos k u+q_{k+h} \sin k u
$$

where $h$ is the number of harmonics. Thus, we have $\mathbf{q}=\left(q_{0}, \cdots, q_{2 h}\right)$.

To derive the evolution equation of $\mathbf{q}$, the problem is set as the minimization of the curve energy $E(\mathbf{q})$ according to the formalism of Lagrangian mechanics and considering $\mathbf{q}$ as the generalized coordinates of the system. To take into account possible high values for $h$ (when complex shapes are considered), we introduce the classical regularization terms to limit the length and the curvature of $\mathcal{C}_{\mathbf{q}}[9]$. In addition, we introduce a third term to ensure the convergence of the curve to the contour even if its initialization is far from it. Several approaches have been proposed to cope with this problem including pressure forces (or balloon forces) [16] or methods based on the gradient vector flow (GVF) fields [12]. 
However, since in our case the surface inside the curve can be easily computed thanks to the Green-Riemann well-known formula: $S_{\mathbf{q}}=\pi\left(q_{0}^{2}+\frac{1}{2} \sum_{k} q_{k}^{2}\right)$, we consider a surface energy term that has to be minimized (when considering an initial curve outside the contour as shown on Fig. 2(a)). In fact, this way to proceed is similar to the use of pressures forces computed all along $\mathcal{C}_{\mathbf{q}}$ as pointed out in [16]. The main advantage is that we obtain here a close form formulation. In that case, we obtain the following equation evolution for a massless curve embedded in a viscous medium:

$$
\mathbf{C} \dot{\mathbf{q}}+\mathbf{K q}=\mathbf{Q}(\mathbf{q})
$$

The vector $\mathbf{Q}(\mathbf{q})$ describes the generalized forces, the matrix $\mathbf{C}$ describes the contribution of the viscous terms while matrix $\mathbf{K}$ includes the contribution to the internal and surface energies. Note that, thanks to the way we parameterized $\mathcal{C}_{\mathbf{q}}$ (see (4) and (5)), these matrices are very simple since they are diagonal and constant. Thus, they can be computed off-line. As a generalized force, we only consider the one due to the contour energy:

$$
E_{\text {cont }}=-\int_{\mathcal{C}_{\mathbf{q}}}\|\nabla I(\mathbf{x}(u))\| d u
$$

where $I(\mathbf{x}(u))$ described the intensity at point $\mathbf{x}(u)$. Indeed, with a fast image processing, the difference between two consecutive images are low and using (7) leads to the convergence of the curve to the contour as proved by the convergence of the control law (see Fig. 2(e) and Fig. 3(d)). The robustness against a large error of the initial curve is shown on Fig. 2 (a).

\section{Results and Discussion}

Image processing and control law computation were performed in real time at 25 frames/second on a PC equipped with a $3 \mathrm{GHz}$ Dual core Xeon Intel processor running Linux. The object 3D parameters involved in the interaction matrix were roughly estimated from pre-operative images in order to fit an ellipsoid object due to the likeness of this shape to usual tumors.

\subsection{Simulation Results with Realistic US Images}

We developed an US software simulator that provides realistic US images of an egg-shaped object. This is useful for testing the active snake algorithm to extract the section contour as it is necessary on real US images. This simulator allows to position and move a $2 \mathrm{D}$ virtual probe on a volume which is composed from a set of parallel real B-scan images. The control gain $\lambda$ was set to 0.3. The results are depicted in Fig. 2. The visual features errors converge to zero roughly exponentially. This validates the method on an object having a different shape than an exact ellipsoid and therefore shows the robustness to object modeling error and measurement perturbations due to discontinuity in the edge detection. 

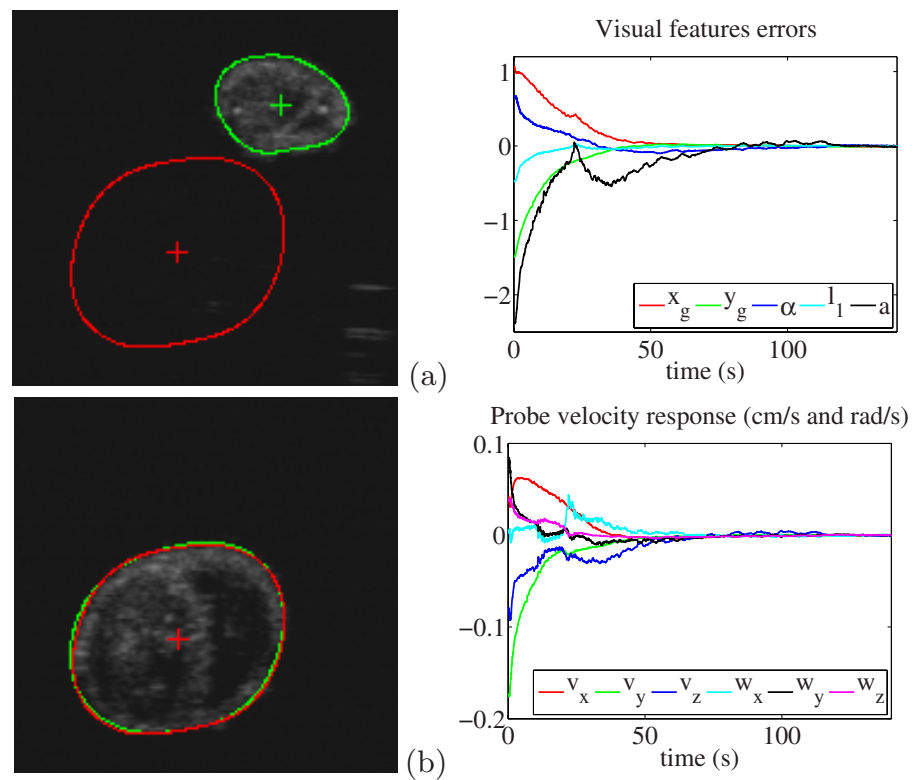

(a)

Probe velocity response $(\mathrm{cm} / \mathrm{s}$ and $\mathrm{rad} / \mathrm{s})$

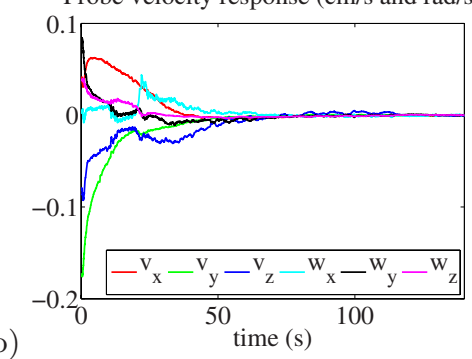

Fig. 3. Results from ex-vivo experiment: (a) Initial (green) and desired (red) image sections edges before applying visual servoing - (b) Current (green) and desired (red) after applying visual servoing (c) Visual error response $\left(\mathrm{cm}, \mathrm{cm}, \mathrm{rad}, \mathrm{cm}\right.$ and $\left.\mathrm{cm}^{2}\right)$ (d) Velocity applied to the probe

\subsection{Ex-vivo Experimental Results}

Finally we test the method during ex-vivo experiments where a 6-DOF medical robot arm similar to the Hippocrate system [17] is used to actuate a 5-10 $\mathrm{MHz}$ linear US probe transducer as shown in Fig. 1(b). The experiment is performed using a motionless rabbit heart suspended in a water-filled box by two nylon yarns. The gain $\lambda$ is set to 0.07 . The experimental results are depicted in Fig. 3. The visual features errors converge to zero and the reached US image corresponds to the desired one as we can see in Fig. 3(b). Note that despite of the difference between the shape of the rabbit heart and the theoretical ellipsoidal model considered in the computation of $\mathbf{L}_{\mathbf{s}}$, the proposed visual servoing succeeds with correct behaviour. Moreover the method has the advantage to be very robust to noise and large initial errors in the image as we can see in Fig [3(a) where initial features are very far from the desired ones.

The successful results obtained with an egg-shaped object and a motionless rabbit heart demonstrated the validity of the approach an its robustness to modelling errors. We currently investigate on the optimal combination of moments which will result in a higher interaction matrix decoupling for better performance. Future work will concern the improvements of the method for more complex-shaped objects that most geometrically fit the organ of interest with online 3D parameters estimation. We also plan to extend the method in order to take into account moving soft tissue. 


\section{References}

1. Abolmaesumi, P., Salcudean, S.E., Zhu, W.-H., Sirouspour, M.R.: Image-guided control of a robot for medical ultrasound. IEEE Trans. on Robotics 18(1), 11-23 (2002)

2. Nelson, T.R., Pretorius, D.H.: Three-dimensional ultrasound imaging. Ultrasound in Medicine and Biol. 24(9), 1243-1270 (1998)

3. Hong, J., Dohi, T., Hashizume, M., Konishi, K., Hata, N.: An ultrasound-driven needle insertion robot for percutaneous cholecystostomy. Physics in Medicine and Biology 49(3), 441-455 (2004)

4. Vitrani, M.A., Morel, G., Bonnet, N., Karouia, M.: A robust ultrasound-based visual servoing approach for automatic guidance of a surgical instrument with in vivo experiments. In: Biomedical Robotics and Biomecatronics BioRob, The first IEEE RAS/EMBS, pp. 35-40 (February 2006)

5. Bachta, A., Krupa, A.: Towards ultrasound image-based visual servoing. In: IEEE Int. Conf. on Robotics and Automation, ICRA 2006, pp. 4112-4117 (May 2006)

6. Krupa, A., Fichtinger, G., Hager, G.D.: Real-time tissue tracking with b-mode ultrasound using speckle and visual servoing. In: Ayache, N., Ourselin, S., Maeder, A. (eds.) MICCAI 2007, Part II. LNCS, vol. 4792, pp. 1-8. Springer, Heidelberg (2007)

7. Espiau, B., Chaumette, F., Rives, P.: A new approach to visual servoing in robotics. IEEE Trans. on Robotics and Automation 8(3), 313-326 (1992)

8. Mebarki, R., Krupa, A., Chaumette, F.: Image moments-based ultrasound visual servoing. In: IEEE int. Conf. on Robotics and Automation ICRA 2008 (May 2008)

9. Kass, M., Witkin, A., Terzopoulos, D.: Snakes: Active contour models. Int. Journal of Computer Vision 1(4), 321-331 (1987)

10. Caselles, V., Kimmel, R., Sapiro, G.: Geodesic active contours. Int. Journal of Computer Vision 22(1), 61-79 (1997)

11. Malladi, R., Sethian, J., Vemuri, B.: Shape modeling with front propagation: A level set approach. IEEE Trans. on Pattern Analysis and Machine Intelligence 17(2), 158-175 (1995)

12. Xu, C., Prince, L.: Snakes, shapes, and gradient vector flow. IEEE Trans. on Image Processing 7, 359-369 (1998)

13. Tauber, C., Batatia, H., Morin, G., Ayache, A.: Robust b-spline snakes for ultrasound image segmentation. IEEE Computers in Cardiology 31, 325-328 (2004)

14. Tang, J., Millington, S., Acton, S., Crandall, J., Hurwitz, S.: Surface extraction and thickness measurement of the articular cartilage from $\mathrm{mr}$ images using directional gradient vector flow snakes. IEEE Trans. on Biomedical Engineering 53(5), 896-906 (2006)

15. Precioso, F., Barlaud, M., Blu, T., Unser, M.: Robust real-time segmentation of images and videos using a smoothing-spline snake-based algorithm. IEEE Trans. on Image Processing (2005)

16. Cohen, L., Cohen, I.: Finite-element methods for active contour models and balloons for 2-d and 3-d images. IEEE Trans. on Pattern Analysis and Machine Intelligence 15(11), 1131-1147 (1993)

17. Pierrot, F., Dombre, E., Degoulange, E., Urbain, L., Caron, P., Boudet, S., Gariepy, J., Megnien, J.: Hippocrate: A safe robot arm for medical applications with force feedback. Medical Image Analysis (MedIA) 3(3), 285-300 (1999) 